International Mathematical Forum, 2, 2007, no. 21, 1039 - 1052

\title{
Subordination and Superordination Results for Certain Subclasses of Analytic Functions
}

\author{
T. N. Shanmugam \\ Department of Information Technology \\ Salalah College of Technology \\ P.O. Box 608 Salalah, Sultanate of Oman \\ S. Sivasubramanian \\ Department of Mathematics \\ Easwari Engineering college \\ Ramapuram, Chennai 600 089, India
}

\author{
M. Darus \\ School of Mathematical Sciences \\ Faculty of Sciences and Technology \\ UKM, Banki 43600, Malaysia \\ C. Ramachandran \\ Department of Mathematics \\ Easwari Engineering college \\ Ramapuram, Chennai 600 089, India
}

\begin{abstract}
The purpose of this present paper is to derive some subordination and superordination results for certain normalized analytic functions in the open unit disk. Relevant connections of the results, which are presented in the paper, with various known results are also considered.
\end{abstract}

Mathematics Subject Classification: Primary 30C80, Secondary 30C45

Keywords: Differential Subordinations, Differential Superordinations, Dominant, Subordinant. 


\section{Introduction}

Let $\mathcal{H}$ be the class of functions analytic in

$$
\Delta:=\{z:|z|<1\}
$$

and $\mathcal{H}[a, n]$ be the subclass of $\mathcal{H}$ consisting of functions of the form

$$
f(z)=a+a_{n} z^{n}+a_{n+1} z^{n+1}+\cdots .
$$

Let $\mathcal{A}$ be the subclass of $\mathcal{H}$ consisting of functions of the form

$$
f(z)=z+a_{2} z^{2}+\cdots .
$$

With a view to recalling the principle of subordination between analytic functions, let the functions $f$ and $g$ be analytic in $\Delta$. Then we say that the function $f$ is subordinate to $g$ if there exists a Schwarz function $\omega(z)$, analytic in $\Delta$ with

$$
\omega(0)=0 \quad \text { and } \quad|\omega(z)|<1 \quad(z \in \Delta)
$$

such that

$$
f(z)=g(\omega(z)) \quad(z \in \Delta)
$$

We denote this subordination by

$$
f \prec g \quad \text { or } \quad f(z) \prec g(z) \quad(z \in \Delta) .
$$

In particular, if the function $g$ is univalent in $\Delta$, the above subordination is equivalent to

$$
f(0)=g(0) \quad \text { and } \quad f(\Delta) \subset g(\Delta) .
$$

Let $p, h \in \mathcal{H}$ and let $\phi(r, s, t ; z): \mathcal{C}^{3} \times \Delta \rightarrow \mathcal{C}$, where $\mathcal{C}$ denotes the set of all complex numbers. If $p$ and $\phi\left(p(z), z p^{\prime}(z), z^{2} p^{\prime \prime}(z) ; z\right)$ are univalent and if $p$ satisfies the second order superordination

$$
h(z) \prec \phi\left(p(z), z p^{\prime}(z), z^{2} p^{\prime \prime}(z) ; z\right),
$$

then $p$ is a solution of the differential superordination (1). (If $f$ is subordinate to $F$, then $F$ is superordinate to $f$.) An analytic function $q$ is called a subordinant if $q \prec p$ for all $p$ satisfying (1). A univalent subordinant $\widetilde{q}$ that satisfies $q \prec \widetilde{q}$ for all subordinants $q$ of (1) is said to be the best subordinant. Recently Miller and Mocanu[5] obtained conditions on $h, q$ and $\phi$ for which the following implication holds:

$$
h(z) \prec \phi\left(p(z), z p^{\prime}(z), z^{2} p^{\prime \prime}(z) ; z\right) \Rightarrow q(z) \prec p(z) .
$$


Using the results of Miller and Mocanu[5], Bulboacă [3] considered certain classes of first order differential superordinations as well as superordinationpreserving integral operators [2]. Ali et al. [1] have used the results of Bulboacă [3] and obtained sufficient conditions for certain normalized analytic functions $f(z)$ to satisfy

$$
q_{1}(z) \prec \frac{z f^{\prime}(z)}{f(z)} \prec q_{2}(z)
$$

where $q_{1}$ and $q_{2}$ are given univalent functions in $\Delta$ with $q_{1}(0)=1$ and $q_{2}(0)=1$. Shanmugam et al. [8] obtained sufficient conditions for a normalized analytic functions $f(z)$ to satisfy

$$
q_{1}(z) \prec \frac{f(z)}{z f^{\prime}(z)} \prec q_{2}(z)
$$

and

$$
q_{1}(z) \prec \frac{z^{2} f^{\prime}(z)}{\{f(z)\}^{2}} \prec q_{2}(z) .
$$

where $q_{1}$ and $q_{2}$ are given univalent functions in $\Delta$ with $q_{1}(0)=1$ and $q_{2}(0)=1$.

Liu [6] introduced and studied the class of functions $B(\lambda, \alpha, \rho)$ defined by $f \in B(\lambda, \alpha, \rho)$ if and only if

$$
\Re\left\{(1-\lambda)\left(\frac{f(z)}{z}\right)^{\alpha}+\lambda \frac{z f^{\prime}(z)}{f(z)}\left(\frac{f(z)}{z}\right)^{\alpha}\right\}>\rho,
$$

where $\lambda \geq 0, \alpha>0$ and $\rho \geq 0$.

The main object of the present sequel to the aforementioned works is to apply a method based on the differential subordination in order to derive several subordination and superordination results. Furthermore, we obtain the previous results of Srivastava and Lashin [9] and Obradovic and Owa as special cases of some of the results presented here.

\section{Preliminaries}

In our present investigation, we shall need the following definition and results.

Definition 2.1 [5, Definition 2, p. 817] Denote by Q, the set of all functions $f(z)$ that are analytic and injective on $\bar{\Delta}-E(f)$, where

$$
E(f)=\left\{\zeta \in \partial \Delta: \lim _{z \rightarrow \zeta} f(z)=\infty\right\}
$$

and are such that $f^{\prime}(\zeta) \neq 0$ for $\zeta \in \partial \Delta-E(f)$. 
Lemma 2.2 [4, Theorem 3.4h, p. 132] Let $q(z)$ be univalent in the unit disk $\Delta$ and $\theta$ and $\phi$ be analytic in a domain $D$ containing $q(\Delta)$ with $\phi(w) \neq 0$ when $w \in q(\Delta)$. Set $Q(z)=z q^{\prime}(z) \phi(q(z))$, $h(z)=\theta(q(z))+Q(z)$. Suppose that

1. $Q(z)$ is starlike univalent in $\Delta$, and

2. $\Re \frac{z h^{\prime}(z)}{Q(z)}>0$ for $z \in \Delta$.

If

$$
\theta(p(z))+z p^{\prime}(z) \phi(p(z)) \prec \theta(q(z))+z q^{\prime}(z) \phi(q(z)),
$$

then $p(z) \prec q(z)$ and $q(z)$ is the best dominant.

Lemma 2.3 [8] Let $q$ be a convex univalent function in $\Delta$. and $\psi, \gamma \in \mathcal{C}$

$\Re\left\{1+\frac{z q^{\prime \prime}(z)}{q^{\prime}(z)}+\frac{\psi}{\gamma}\right\}>0$. If $p(z)$ is analytic in $\Delta$ and

$$
\psi p(z)+\gamma z p^{\prime}(z) \prec \psi q(z)+\gamma z q^{\prime}(z)
$$

then $p(z) \prec q(z)$ and $q$ is the best dominant.

Lemma 2.4 [3] Let $q(z)$ be convex univalent in the unit disk $\Delta$ and $\vartheta$ and $\varphi$ be analytic in a domain $D$ containing $q(\Delta)$. Suppose that

1. $\Re\left[\vartheta^{\prime}(q(z)) / \varphi(q(z))\right]>0$ for $z \in \Delta$,

2. $z q^{\prime}(z) \varphi(q(z))$ is starlike univalent in $\Delta$.

If $p(z) \in \mathcal{H}[q(0), 1] \cap Q$, with $p(\Delta) \subseteq D$, and $\vartheta(p(z))+z p^{\prime}(z) \varphi(p(z))$ is univalent in $\Delta$, and

$$
\vartheta(q(z))+z q^{\prime}(z) \varphi(q(z)) \prec \vartheta(p(z))+z p^{\prime}(z) \varphi(p(z))
$$

then $q(z) \prec p(z)$ and $q$ is the best subordinant.

Lemma 2.5 [5, Theorem 8, p. 822] Let $q$ be convex univalent in $\Delta$ and $\gamma \in \mathcal{C}$. Further assume that $\Re[\bar{\gamma}]>0$. If $p(z) \in \mathcal{H}[q(0), 1] \cap Q, p(z)+\gamma z p^{\prime}(z)$ is univalent in $\Delta$, then

$$
q(z)+\gamma z q^{\prime}(z) \prec p(z)+\gamma z p^{\prime}(z)
$$

implies $q(z) \prec p(z)$ and $q$ is the best subordinant. 


\section{Subordination for Analytic Functions}

By using Lemma 2.3, we first prove the following.

Theorem 3.1 Let $q$ be univalent in $\Delta, \lambda \geq 0$ and $\alpha>0$. Suppose $q$ satisfies

$$
\Re\left\{1+\frac{z q^{\prime \prime}(z)}{q^{\prime}(z)}\right\}+\frac{\lambda}{\alpha}>0 .
$$

If $f \in \mathcal{A}$ satisfies the subordination:

$$
(1-\lambda)\left(\frac{f(z)}{z}\right)^{\alpha}+\lambda \frac{z f^{\prime}(z)}{f(z)}\left(\frac{f(z)}{z}\right)^{\alpha} \prec q(z)+\frac{\lambda z q^{\prime}(z)}{\alpha}
$$

then

$$
\left(\frac{f(z)}{z}\right)^{\alpha} \prec q(z)
$$

and $q$ is the best dominant.

Proof. Define the function $p(z)$ by

$$
p(z):=\left(\frac{f(z)}{z}\right)^{\alpha} .
$$

Then

$$
\frac{z p^{\prime}(z)}{p(z)}=\alpha\left[\frac{z f^{\prime}(z)}{f(z)}-1\right]
$$

which, in light of hypothesis (10) of Theorem 3.1, yields the following subordination

$$
p(z)+\frac{\lambda z p^{\prime}(z)}{\alpha} \prec q(z)+\frac{\lambda z q^{\prime}(z)}{\alpha} .
$$

The assertion (10) of Theorem 3.1 now follows by an application of Lemma 2.3 with $\gamma=\frac{\lambda}{\alpha}$ and $\psi=1$.

Taking $q(z)=\frac{1+A z}{1+B z}$ in Theorem 3.1, we have the following corollary.

Corollary 3.2 Let $-1 \leq B<A \leq 1$ and (3) hold. If $f \in \mathcal{A}$, and

$$
(1-\lambda)\left(\frac{f(z)}{z}\right)^{\alpha}+\lambda \frac{z f^{\prime}(z)}{f(z)}\left(\frac{f(z)}{z}\right)^{\alpha} \prec \frac{\lambda(A-B) z}{\alpha(1+B z)^{2}}+\frac{1+A z}{1+B z},
$$

then

$$
\left(\frac{f(z)}{z}\right)^{\alpha} \prec \frac{1+A z}{1+B z}
$$

and $\frac{1+A z}{1+B z}$ is the best dominant. 
Our Theorem 3.1 for the choice of $q(z)=\frac{1+z}{1-z}$, reduces to

Corollary 3.3 Let (3) hold. If $f \in \mathcal{A}$, and

$$
(1-\lambda)\left(\frac{f(z)}{z}\right)^{\alpha}+\lambda \frac{z f^{\prime}(z)}{f(z)}\left(\frac{f(z)}{z}\right)^{\alpha} \prec \frac{2 \lambda z}{\alpha(1-z)^{2}}+\frac{1+z}{1-z},
$$

then

$$
\left(\frac{f(z)}{z}\right)^{\alpha} \prec \frac{1+z}{1-z}
$$

and $\frac{1+z}{1-z}$ is the best dominant.

Theorem 3.4 Let $q$ be univalent in $\Delta, \alpha, \gamma \in \mathcal{C}$ and $0 \leq \beta \leq 1$. Let $f \in \mathcal{A}$. Suppose $q$ satisfies

$$
\Re\left\{1+\frac{z q^{\prime \prime}(z)}{q^{\prime}(z)}-\frac{z q^{\prime}(z)}{q(z)}\right\}>0 .
$$

If

$$
1+\gamma \alpha\left[\frac{z f^{\prime}(z)}{f(z)}-1\right] \prec 1+\gamma \frac{z q^{\prime}(z)}{q(z)}
$$

then

$$
\left(\frac{f(z)}{z}\right)^{\alpha} \prec q(z)
$$

and $q$ is the best dominant.

Proof. Define the function $p(z)$ by

$$
p(z):=\left[\frac{f(z)}{z}\right]^{\alpha}, z \neq 0
$$

Then a computation shows that

$$
\alpha\left\{\frac{z f^{\prime}(z)}{f(z)}-1\right\}=\frac{z p^{\prime}(z)}{p(z)}
$$

By setting

$$
\theta(\omega):=1, \quad \text { and } \quad \phi(\omega):=\frac{\gamma}{\omega}
$$


it can be easily observed that $\theta(\omega)$ is analytic in $\mathcal{C}, \phi(\omega)$ is analytic in $\mathcal{C} \backslash\{0\}$ and that

$$
\phi(\omega) \neq 0 \quad(\omega \in \mathcal{C} \backslash\{0\}) .
$$

Also, we let

$$
Q(z)=z q^{\prime}(z) \phi(q(z))=\gamma \frac{z q^{\prime}(z)}{q(z)}
$$

and

$$
h(z)=\theta\{q(z)\}+Q(z)=1+\gamma \frac{z q^{\prime}(z)}{q(z)} .
$$

From (5), we find that $Q(z)$ is starlike univalent in $\Delta$ and that

$$
\Re\left(\frac{z h^{\prime}(z)}{Q(z)}\right)=\Re\left\{1+\frac{z q^{\prime \prime}(z)}{q^{\prime}(z)}-\frac{z q^{\prime}(z)}{q(z)}\right\}>0,
$$

by the hypothesis (5) of Theorem 3.4. Thus, by applying Lemma 2.2, our proof of Theorem 3.4 is completed.

For a special case when $q(z)=\frac{1}{(1-z)^{2 b}} \quad(b \in \mathcal{C} \backslash\{0\}), \quad \gamma=\frac{1}{b}$ and $\alpha=$ 1, Theorem 3.4 reduces at once to the following known result obtained by Srivastava and Lashin [9].

Corollary 3.5 Let $b$ be a non zero complex number. If $f \in \mathcal{A}$, and

$$
1+\frac{1}{b}\left[\frac{z f^{\prime}(z)}{f(z)}-1\right] \prec \frac{1+z}{1-z}
$$

then

$$
\frac{f(z)}{z} \prec \frac{1}{(1-z)^{2 b}}
$$

and $\frac{1}{(1-z)^{2 b}}$ is the best dominant.

For $q(z)=(1+B z)^{\frac{\mu(A-B)}{B}}, \alpha=\mu, \gamma=\frac{1}{\alpha}$ in Theorem 3.4, we get the following known result obtained by Obradović and Owa [7].

Corollary 3.6 Let $-1 \leq B<A \leq 1$. Let $\mu, A, B$ satisfy the relation either

$$
\left|\frac{\mu(A-B)}{B}-1\right| \leq 1
$$

or

$$
\left|\frac{\mu(A-B)}{B}+1\right| \leq 1
$$


If $f \in \mathcal{A}$, and

$$
\frac{z f^{\prime}(z)}{f(z)} \prec \frac{1+A z}{1+B z}
$$

then

$$
\left(\frac{f(z)}{z}\right)^{\mu} \prec(1+B z)^{\frac{\mu(A-B)}{B}} \quad(z \in \Delta ; z \neq 0 ; \mu \in \mathcal{C} ; \mu \neq 0)
$$

and $(1+B z)^{\frac{\mu(A-B)}{B}}$ is the best dominant.

Theorem 3.7 Let q be univalent in $\Delta$. Suppose q satisfies

$$
\Re\left\{1+q(z)+\frac{z q^{\prime \prime}(z)}{q^{\prime}(z)}-\frac{z q^{\prime}(z)}{q(z)}\right\}>0 .
$$

If $f \in \mathcal{A}$ satisfies the subordination:

$$
\frac{\lambda z^{3} f^{\prime \prime \prime}(z)+(1+2 \lambda) z^{2} f^{\prime \prime}(z)+z f^{\prime}(z)}{\lambda z^{2} f^{\prime \prime}(z)+z f^{\prime}(z)} \prec q(z)+\frac{z q^{\prime}(z)}{q(z)},
$$

then

$$
\frac{\lambda z^{2} f^{\prime \prime}(z)+z f^{\prime}(z)}{(1-\lambda) f(z)+\lambda z f^{\prime}(z)} \prec q(z)
$$

and $q$ is the best dominant.

Proof. Define the function $p(z)$ by

$$
p(z):=\frac{\lambda z^{2} f^{\prime \prime}(z)+z f^{\prime}(z)}{(1-\lambda) f(z)+\lambda z f^{\prime}(z)} .
$$

Then

$$
\frac{z p^{\prime}(z)}{p(z)}=\frac{\lambda z^{3} f^{\prime \prime \prime}(z)+(1+2 \lambda) z^{2} f^{\prime \prime}(z)+z f^{\prime}(z)}{\lambda z^{2} f^{\prime \prime}(z)+z f^{\prime}(z)}-\frac{\lambda z^{2} f^{\prime \prime}(z)+z f^{\prime}(z)}{(1-\lambda) f(z)+\lambda z f^{\prime}(z)}
$$

which, in light of hypothesis (10) of Theorem 3.7, yields the following subordination

$$
p(z)+\frac{z p^{\prime}(z)}{p(z)} \prec q(z)+\frac{z q^{\prime}(z)}{q(z)} .
$$

By setting

$$
\theta(\omega):=\omega, \quad \text { and } \quad \phi(\omega):=\frac{1}{\omega},
$$


it can be easily observed that $\theta(\omega)$ is analytic in $\mathcal{C}, \phi(\omega)$ is analytic in $\mathcal{C} \backslash\{0\}$ and that

$$
\phi(\omega) \neq 0 \quad(\omega \in \mathcal{C} \backslash\{0\})
$$

Also, we let

$$
Q(z)=z q^{\prime}(z) \phi(q(z))=\frac{z q^{\prime}(z)}{q(z)}
$$

and

$$
h(z)=\theta\{q(z)\}+Q(z)=q(z)+\frac{z q^{\prime}(z)}{q(z)} .
$$

From (9), we find that $Q(z)$ is starlike univalent in $\Delta$ and that

$$
\Re\left(\frac{z h^{\prime}(z)}{Q(z)}\right)=\Re\left\{1+q(z)+\frac{z q^{\prime \prime}(z)}{q^{\prime}(z)}-\frac{z q^{\prime}(z)}{q(z)}\right\}>0,
$$

by the hypothesis (9) of Theorem 3.7. Thus, by applying Lemma 2.2, our proof of Theorem 3.7 is completed.

\section{Superordination for analytic functions}

Next, applying Lemma 2.5, we obtain the following two theorems.

Theorem 4.1 Let $q$ be convex univalent in $\Delta, \lambda \geq 0$ and $\alpha>0$ and $\left(\frac{f(z)}{z}\right)^{\alpha} \in \mathcal{H}[q(0), 1] \cap Q$. Let

$$
(1-\lambda)\left(\frac{f(z)}{z}\right)^{\alpha}+\lambda \frac{z f^{\prime}(z)}{f(z)}\left(\frac{f(z)}{z}\right)^{\alpha}
$$

be univalent in $\Delta$. If $f \in \mathcal{A}$ satisfies the superordination:

$$
q(z)+\frac{\lambda z q^{\prime}(z)}{\alpha} \prec(1-\lambda)\left(\frac{f(z)}{z}\right)^{\alpha}+\lambda \frac{z f^{\prime}(z)}{f(z)}\left(\frac{f(z)}{z}\right)^{\alpha}
$$

then

$$
q(z) \prec\left(\frac{f(z)}{z}\right)^{\alpha}
$$

and $q$ is the best subordinant. 
Proof. Define the function $p(z)$ by

$$
p(z):=\left(\frac{f(z)}{z}\right)^{\alpha} .
$$

Then a computation shows that

$$
\alpha\left\{\frac{z f^{\prime}(z)}{f(z)}-1\right\}=\frac{z p^{\prime}(z)}{p(z)}
$$

Theorem 4.1 follows as an application of Lemma 2.5.

Taking $q(z)=\frac{1+A z}{1+B z}$ in Theorem 4.1, we get the following corollary.

Corollary 4.2 Let $-1 \leq B<A \leq 1$. Let $q$ be convex univalent in $\Delta$. Suppose $\left(\frac{f(z)}{z}\right)^{\alpha} \in \mathcal{H}[q(0), 1] \cap Q$ and

$$
(1-\lambda)\left(\frac{f(z)}{z}\right)^{\alpha}+\lambda \frac{z f^{\prime}(z)}{f(z)}\left(\frac{f(z)}{z}\right)^{\alpha}
$$

be univalent in $\Delta$. If

$$
\frac{\lambda(A-B) z}{\alpha(1+B z)^{2}}+\frac{1+A z}{1+B z} \prec(1-\lambda)\left(\frac{f(z)}{z}\right)^{\alpha}+\lambda \frac{z f^{\prime}(z)}{f(z)}\left(\frac{f(z)}{z}\right)^{\alpha},
$$

then

$$
\frac{1+A z}{1+B z} \prec\left(\frac{f(z)}{z}\right)^{\alpha}
$$

and $\frac{1+A z}{1+B z}$ is the best subordinant.

Theorem 4.3 Let $q$ be convex univalent in $\Delta, \lambda \geq 0$ and $\alpha>0$ and $\left(\frac{f(z)}{z}\right)^{\alpha} \in \mathcal{H}[q(0), 1] \cap Q$. Let $f \in \mathcal{A}$. If

$$
1+\gamma \frac{z q^{\prime}(z)}{q(z)} \prec 1+\gamma \alpha\left[\frac{z f^{\prime}(z)}{f(z)}-1\right]
$$

then

$$
q(z) \prec\left(\frac{f(z)}{z}\right)^{\alpha}
$$

and $q$ is the best subordinant. 
Theorem 4.4 Let $q$ be univalent in $\Delta$. Suppose $q$ satisfies

$$
\Re\left\{q(z) q^{\prime}(z)\right\}>0 .
$$

If $f \in \mathcal{A}$ satisfies the superordination:

$$
q(z)+\frac{z q^{\prime}(z)}{q(z)} \prec \frac{\lambda z^{3} f^{\prime \prime \prime}(z)+(1+2 \lambda) z^{2} f^{\prime \prime}(z)+z f^{\prime}(z)}{\lambda z^{2} f^{\prime \prime}(z)+z f^{\prime}(z)},
$$

then

$$
q(z) \prec \frac{\lambda z^{2} f^{\prime \prime}(z)+z f^{\prime}(z)}{(1-\lambda) f(z)+\lambda z f^{\prime}(z)}
$$

and $q$ is the best subordinant.

\section{Sandwich results}

Combining the results of differential subordination and superordination, we state the following "sandwich results":

Theorem 5.1 Let $q_{1}$ be convex univalent and $q_{2}$ be univalent in $\Delta, \lambda \geq 0$, and $\alpha>0$ and $q_{2}$ satisfies (3). Suppose

$$
\left(\frac{f(z)}{z}\right)^{\alpha} \in \mathcal{H}[q(0), 1] \cap Q
$$

and

$$
(1-\lambda)\left(\frac{f(z)}{z}\right)^{\alpha}+\lambda \frac{z f^{\prime}(z)}{f(z)}\left(\frac{f(z)}{z}\right)^{\alpha}
$$

is univalent in $\Delta$. If $f \in \mathcal{A}$ satisfies

$$
q_{1}(z)+\frac{\lambda z q_{1}^{\prime}(z)}{\alpha} \prec(1-\lambda)\left(\frac{f(z)}{z}\right)^{\alpha}+\lambda \frac{z f^{\prime}(z)}{f(z)}\left(\frac{f(z)}{z}\right)^{\alpha} \prec q_{2}(z)+\frac{\lambda}{\alpha} z q_{2}^{\prime}(z)
$$

then

$$
q_{1}(z) \prec\left(\frac{f(z)}{z}\right)^{\alpha} \prec q_{2}(z)
$$

and $q_{1}$ and $q_{2}$ are respectively the best subordinant and best dominant. 
Theorem 5.2 Let $q_{1}$ be convex univalent and $q_{2}$ be univalent in $\Delta, \lambda \geq 0$, and $\alpha>0$ and $q_{2}$ satisfies (5). Suppose

$$
\left(\frac{f(z)}{z}\right)^{\alpha} \in \mathcal{H}[q(0), 1] \cap Q
$$

and

$$
(1-\lambda)\left(\frac{f(z)}{z}\right)^{\alpha}+\lambda \frac{z f^{\prime}(z)}{f(z)}\left(\frac{f(z)}{z}\right)^{\alpha}
$$

is univalent in $\Delta$. If $f \in \mathcal{A}$ satisfies

$$
1+\gamma \frac{z q_{1}^{\prime}(z)}{q_{1}(z)} \prec(1-\lambda)\left(\frac{f(z)}{z}\right)^{\alpha}+\lambda \frac{z f^{\prime}(z)}{f(z)}\left(\frac{f(z)}{z}\right)^{\alpha} \prec 1+\gamma \frac{z q_{2}^{\prime}(z)}{q_{2}(z)},
$$

then

$$
q_{1}(z) \prec\left(\frac{f(z)}{z}\right)^{\alpha} \prec q_{2}(z)
$$

and $q_{1}$ and $q_{2}$ are respectively the best subordinant and best dominant.

Theorem 5.3 Let $q_{1}$ be convex univalent and $q_{1}$ be univalent in $\Delta$. Suppose $q_{1}$ satisfies (15) and $q_{2}$ satisfies (9). Suppose

$$
\frac{\lambda z^{2} f^{\prime \prime}(z)+z f^{\prime}(z)}{(1-\lambda) f(z)+\lambda z f^{\prime}(z)} \in \mathcal{H}[q(0), 1] \cap Q,
$$

and

$$
\frac{\lambda z^{3} f^{\prime \prime \prime}(z)+(1+2 \lambda) z^{2} f^{\prime \prime}(z)+z f^{\prime}(z)}{\lambda z^{2} f^{\prime \prime}(z)+z f^{\prime}(z)}
$$

is univalent in $\Delta$. If $f \in \mathcal{A}$ satisfies the superordination:

$$
q_{1}(z)+\frac{z q_{1}^{\prime}(z)}{q_{1}(z)} \prec \frac{\lambda z^{3} f^{\prime \prime \prime}(z)+(1+2 \lambda) z^{2} f^{\prime \prime}(z)+z f^{\prime}(z)}{\lambda z^{2} f^{\prime \prime}(z)+z f^{\prime}(z)} \prec q_{2}(z)+\frac{z q_{2}^{\prime}(z)}{q_{2}(z)},
$$

then

$$
q_{1}(z) \prec \frac{\lambda z^{2} f^{\prime \prime}(z)+z f^{\prime}(z)}{(1-\lambda) f(z)+\lambda z f^{\prime}(z)} \prec q_{2}(z)
$$

and $q_{1}$ and $q_{2}$ are respectively the best subordinant and best dominant. 
For $\lambda=0$, we get the following result.

Theorem 5.4 Let $q_{1}$ be convex univalent and $q_{1}$ be univalent in $\Delta, \lambda \geq 0$ and $\alpha>0$. Suppose $q_{1}$ satisfies (15) and $q_{2}$ satisfies (9). Suppose

$$
\frac{z f^{\prime}(z)}{f(z)} \in \mathcal{H}[q(0), 1] \cap Q,
$$

and

$$
1+\frac{z f^{\prime \prime}(z)}{f^{\prime}(z)}
$$

is univalent in $\Delta$. If $f \in \mathcal{A}$ satisfies the superordination:

$$
q_{1}(z)+\frac{z q_{1}^{\prime}(z)}{q_{1}(z)} \prec 1+\frac{z f^{\prime \prime}(z)}{f^{\prime}(z)} \prec q_{2}(z)+\frac{z q_{2}^{\prime}(z)}{q_{2}(z)}
$$

then

$$
q_{1}(z) \prec \frac{z f^{\prime}(z)}{f(z)} \prec q_{2}(z)
$$

and $q_{1}$ and $q_{2}$ are respectively the best subordinant and best dominant.

Acknowledgement The work presented here is partially supported by SAGA(Scientific Advancement Fund Allocation)-STGL-012-2006, Academy of Sciences, Malaysia.

\section{References}

[1] R. M. Ali, V. Ravichandran, M. Hussain Khan and K. G. Subramanian, Differential sandwich theorems for certain analytic functions, Far East J.Math. Sci. (FJMS) 15(1) (2005), 87-94.

[2] T. Bulboacă, A class of superordination-preserving integral operators, Indag. Math. New Ser. 13(3)(2002), 301-311.

[3] T. Bulboacă, Classes of first-order differential superordinations, Demonstr. Math. 35(2) (2002), 287-292.

[4] S. S. Miller, P. T. Mocanu, Differential Subordinations: Theory and Applications. Pure and Applied Mathematics No. 225, Marcel Dekker, New York, (2000). 
[5] S. S. Miller and P. T. Mocanu, Subordinants of differential superordinations, Complex Variables, 48(10) (2003), 815-826.

[6] M. Liu, Properties for some subclasses of analytic functions, Bull. Inst. Math. Acad. Sinica 30(1) (2002), 9-26.

[7] M. Obradović and S. Owa, On certain properties for some classes of starlike functions, J. Math. Anal. Appl. 145 (1990), no. 2, 357-364.

[8] T. N. Shanmugam, V. Ravichandran and S. Sivasubramanian, Differential sandwich theorems for some subclasses of analytic functions, Austral. Math. Anal. Appl. 3(1) (2006), Article 8, 1-11 (electronic).

[9] H. M. Srivastava, and A. Y. Lashin, Some applications of the BriotBouquet differential subordination, JIPAM. J. Inequal. Pure Appl. Math. 6 (2005), no. 2, Article 41, 7 pp.

\section{Received: August 19, 2006}

